\title{
Mapillary based plant distributions of ethnobotanical afforestation.
}

Dr Bheemaiah, Anil Kumar, A.B Seattle W.A 98125

miyawaki@yopmail.com

\begin{abstract}
:
Mapillary is an open-source code base for the use of GPU based Deep Learning for Semantic Segmentation of wild images. We propose the creation of an autonomous drone for the automated capture of scientific images of medicinal and edible plants to create geotagged maps of plants on Mapillary.com with additional tags on plant sizes, species, and edible and medicinal value. This information is used in the planning of sponsored five or more level afforestation as social and academic forestry for edible and medicinal value. The same research is also useful in planning afforestation on Mars.
\end{abstract}

Keywords: Miyawakis, Mapillary, Seamless Segmentation, FPN, ResNet50, Redtail, Edible and Medicinal Plants, Geotag

What:

Mapillary is deep learning, Nvidia inception venture to segment user loaded images and embed into a GIS database, for auto navigation end uses, in this paper we explore plant geotags with ontologies for a database of plant images and distributions for location information of edible and medicinal plants. This service is to be unified with apps like Google Lens and a host of image reverse search engine APIs, to return ontologies of the plant database, including plant density distributions and historical data of distributions, for invasive plant information.

How:

Mapillary uses an open-source code base, there are three tasks involved, with user stories.

1. Addition of search APIs to ROI determination in mask head segmentation phase to add tags with ontologies, like size, species, and other ontologies. 
2. Addition of tags with the addition of search result information and enhancement of the identification algorithm, for size determination and species addition.

3. Modification of automated image sequence capture with improved content sensitivity for IOT, Redtail based drone photography using an Iris or Solo Drone.

\section{Summary:}

\section{Main Points:}

1. Extension of the Redtail architecture for Drones and Rovers, with $6 \mathrm{G}$ integration.

2. ROI based JSON as request to Image Query API, Google and Bing services.

3. Extension of object categories of Mapillary.

\section{Applications:}

Acoustic Proofing and afforestation for natural cover and social ethnobotany. Academic temple, in proof of primary growth as grace in a natural academic spirituality. The true inner sanctum.

Code Base:https://github.com/vedek/mapillaryToDo

\section{Introduction.}

Mapillary is a Nvidia inception venture, which is a portal, with a large user base to upload images of street based and trail based scenes for seamless segmentation to assist in the integration of autonomous navigation and create image datasets with geotag information. We enhance the segmentation categories of object data with plant categorization by size and taxonomy, ontologies and other queries for ethnobotanical and scientific distribution density maps for afforestation activities.

\section{Problem Definition.}

1.Extension of the Redtail architecture for Drones and Rovers, with communication channels [[cn_i]], for mapillary integration, with sensor stream $\mathrm{C}$, with a reactive event stream [[Et, img]] based on FPN derived event triggers. 2. ROI based JSON as request to Image Query API, Google and Bing services, with ontologies mapped to a knowledge graph from a response JSON.

3.Extension of object categories of Mapillary to extend the segmentation of vegetation to [layer, species, taxonomy ontologies \#n] 


\section{Background.}

(Shiferaw, Bewket, and Eckert 2019; Yang, Zheng, and Wang 2019; Baniwal and Jain, n.d.; Hengl et al. 2018; Fielding 1999; Senay and Worner 2019; Remagnino et al. 2016)

Afforestation, has been a primordial human activity, to conserve the natural genomics and genetic sources. Analogous to the resurrection of Christ, as in Christology, the present forests can be seen in the role of Jesus in the 'sacrifice' in the path of development, afforestation using models by Akira Miyawaki, called Miyawaki forests can be thought of as the resurrection in Christology, all based on the conservation of the natural genome.

Miyawakis also build resilience and have many roles, apart from social forestry, a source of edible and medicinal plants and green spaces, to mitigate pollution.

Mapillary an A.I based map of images, automatically collected from automobiles and user contributions, is now extended to drone and aircraft based collection with intelligent event triggers. The semantic segmentation is also improved to add more ontologies to the tagging.

\section{$<$ original-contribution $>$}

\section{Formal Definitions:}

A Miyawaki is defined as a data structure Forest $=\left[\mathrm{T} i \_\mathrm{j}, \mathrm{T} \mathrm{k} \_1, \ldots \mathrm{T} \mathrm{x} \_\mathrm{y}\right]$

Where $\mathrm{T} \mathrm{i} \_\mathrm{j}$ is the $\mathrm{jth}$ tree or plant of layer $\mathrm{i}$. A Miyawaki consists usually of five or more layers.

For each plant $\mathrm{p}$ in a database, we have the following tags.

[Layer, SpeciesData, GeoTag, Ontologies ]
We data mine the natural distribution of a specific species $\mathrm{s}$, from $\mathrm{f}\left(\mathrm{s}, \mathrm{x}^{\wedge}, \mathrm{t}\right)$ and $\mathrm{D}\left(\mathrm{s} \_\mathrm{i}, 1\right)$ for species $\mathrm{s} \_\mathrm{i}$ as the natural distribution of species for a Layer 1 , When a Miyawaki is created, the initial species distribution is matched to the distribution of that region of native species, both edible and medicinal and of ethnobotanical value. $\mathrm{D} 0\left(\mathrm{~s} \_\mathrm{i}, 1\right)$ is the initial species distribution and $\operatorname{Dt}\left(\mathrm{s} \_\mathrm{i}, \mathrm{l}\right)$ is the distribution with time which changes because of the changes in species composition and the arrival or disappearance of species, including invasive spoecies and the sustainable harvesting/foraging by wildlife of plants.

Definition of an invasive species:

Any species s_inv is defined to be invasive if the population density in $\operatorname{Dt}\left(s_{-}\right.$i,l) is out of balance, defined by $\mid \mathbf{s} \_$inv $\mid /\left(\mid \mathbf{s} \_\right.$inv $|+|[$Sum $] \mid$s_i $\left.\mid\right)$being greater than a defined constant which may be geo specific, c_g.

Sustainable harvesting and grazing:

Every plant species is of edible or medicinal use to fauna and humans and the primary purpose of a Miyawaki is to provide natural cover for the fauna there.

if a species s_har is harvested of grazed, then a similar constant c_sus exists which is the lower bound and c_g the upper bound for that species.

Stability Theorem:

$\operatorname{Dt}\left(s \_i, 1\right)$ remains, for every s within the bounds (s_sus, s_inv) for efficient forest_management(). forest_managemnt() is termed "Control Schizophrenia" a term for a curable disorder of humanity in aneed to manage or control a Miyawaki Forest, A Miyawaki is a restoration principle, a natural recovery of which human action is a medium. There is thus no managemnt of the natural order, all afforestation is part of a 
spirituality and not a science of primary growth as recovery of nature after human intervention has ended.

The so called "Forest Management" consists of only of limiting unnatural grazing and over harvesting and decreasing harvesting to restore balance of near invasions which are an indicator of unhealth of a Miyawaki, which is usually impossible, owing to the practised spirituality, the grace of the primary forest.

If due to the actions of nature for any species s_endangered, the population falls below a critical threshold then various measured for the conservation of that species in the Miyawaki have to be undertaken by afforestation of that species in a synergy principle.

Forest Management is thus only a management of Human activities and headstarting as a Miyawaki is a natural primary forest growth in the atomicity of the unmanifested, manifested in headstarting activities and natural recovery.

Synergy Theorem:

The stability of one species $\mathrm{s} \_\mathrm{i}$ is dependant on the $\Sigma \Sigma\left(D\left(s \_i, l\right)\right)$ the 'whole' of inter-species synergies called equilibrium stability in dynamical systems theory.

Thus conservation in Miyawakis is defined in the 'whole' in the interdependence of the layers, the canopy and the undergrowth and in flora-fauna dynamics.

Afforestation as defined by Akira Miyawaki : Formal Definitions.

Mapillary Architecture and User Stories.

\section{Determination of $\mathrm{D}\left(\mathrm{s} \_\mathrm{i}, \mathrm{x}^{\wedge}, \mathrm{I}\right)$}

Architecture:

Data feedback architectures for Mapillary image capture.

Keywords: Seamless Scene Integration, Mask Head, FPN based segmentation, LightNet architecture for segmentation, panoptic segmentation, FPN based data piped to event trigger Et.

Let there exist a streamInput $\mathrm{C},->[\mathrm{C}, \mathrm{E}]$ on [Et]

$\mathrm{Et}=->[\mathrm{C}, \mathrm{FPN}(\mathrm{C})]$

FPN is defined in (Porzi et al)

Optimal ROI for Google Search API or Bing Image API.

ROI from Mask Head, add external API call to Bing or Google Search with optimal ROI image as JSON.(Porzi et al)

Ontology algorithms and knowledge graphs from JSON response.

For JSON Response s,

1. taxonomy construction (ATC)

For Knowledge Data Structures, $\mathrm{K}=\left[\left[\mathrm{v}_{\mathrm{i}} \mathrm{i}\right]\right]$ $\mathrm{O}->\left(\mathrm{v}_{-} \mathrm{i}=[\mathrm{T}]\right)$

Taxonomy Ontology $\mathrm{T}=[$ Species, [Related_Species_i]]

Taxonomy Ontology $\mathrm{T} 1$ =

[Gravitational_Nomenclature, Query]

Taxonomy Ontology T2 $=[$ EthnoBotany $(\mathrm{Eg}$

Midwest druidery), Query]

2. Other Ontologies.

//to be filled later.

Addition of Mapillary to a Redtail based

Drone with sensor stream $\mathrm{C}$ as input and a 
$6 \mathrm{G}$ communication channel [[c_i $]]$ (Neubeur et al)(Nvidia Redtail)

\section{Kanban: (On Taiga)}

User Story \#001 10 hours: Interpolation and determination of species distribution by ROI based segmentation with geo-tags. User Story \#002 15 hours : Feedback from distribution model for input images using Semantic Head. User Story \#003 10 hours : Data Base of images with tag and ontologies data structures for determining, $\mathrm{D}\left(\mathrm{s} \_\mathrm{i}, \mathrm{x}^{\wedge}, 1\right)$.

\section{Story:}

With Himalayan and Artic circle forestry as the Case Studies, automated afforestation is resolved into taskoids which are customizable, in the design of such primary afforestation for natural cover to fauna, to prevent unnecessary disturbance from flying drones, create ethnobotanical sources of medicinal and edible flora and conserve natural genome, we define a formal framework for afforestation based resilience as defined by Akiro Miyawaki, a japanese plant biologist.

\section{$<$ /original-contribution $>$}

\section{Discussion.}

Cartography(Wassermann 1978; Goodrick 1982) is an art and science, and the Earth was discovered to be round as opposed to a
Flat structure through the work of cartographers, the present resolution of cartography and satellite based maps is roughly 15 meter, ("Spatial Resolution of Google Earth Imagery" n.d.) with the passage to time, the resolution would increase to the scale of centimeters, enabling the description of each plant and tree on a GIS service like wikimapia. This paper is on the data mining and the creation of a semantic approach to cartography, geotagged map of human relevant information of objects, in this case flora. We have thus described the technology of drones and other mobility and the automated creation of such cartography.

\section{Future Work.}

Automated linear scanners, similar to the Messier catalog of stars for arboretums and genomic conservatories in the Arctic circle.

\section{References.}

Baniwal, Sachin, and Rachna Jain. n.d. "Machine Learning To Identify Plant: A Review." SSRN Electronic Journal. https://doi.org/10.2139/ssrn.3379060.

Fielding, Alan. 1999. Machine Learning Methods for Ecological Applications. Springer Science \& Business Media.

Goodrick, B. E. 1982. "What Is Cartography?" Cartography. https://doi.org/10.1080/00690805.1982.104 38186.

Hengl, Tomislav, Markus G. Walsh, Jonathan Sanderman, Ichsani Wheeler, Sandy P. 
Harrison, and Iain C. Prentice. 2018.

"Global Mapping of Potential Natural

Vegetation: An Assessment of Machine

Learning Algorithms for Estimating Land

Potential." PeerJ 6 (August): e5457.

Remagnino, Paolo, Simon Mayo, Paul Wilkin, James Cope, and Don Kirkup. 2016.

Computational Botany: Methods for

Automated Species Identification. Springer.

Senay, Senait D., and Susan P. Worner. 2019.

"Multi-Scenario Species Distribution

Modeling." Insects 10 (3).

https://doi.org/10.3390/insects10030065.

Shiferaw, Hailu, Woldeamlak Bewket, and

Sandra Eckert. 2019. "Performances of

Machine Learning Algorithms for Mapping

Fractional Cover of an Invasive Plant

Species in a Dryland Ecosystem." Ecology and Evolution 9 (5): 2562-74.

"Spatial Resolution of Google Earth Imagery."

n.d. Geographic Information Systems Stack Exchange. Accessed August 21, 2019.

https://gis.stackexchange.com/questions/11

395/spatial-resolution-of-google-earth-imag

ery.

Wassermann, W. 1978. "Cartography as a

Science." Cartography.

https://doi.org/10.1080/00690805.1978.104

38010.

Yang, Nan, Zeyu Zheng, and Tianran Wang. 2019. "Model Loss and Distribution Analysis of Regression Problems in Machine Learning." Proceedings of the 2019 11th International Conference on Machine Learning and Computing ICMLC '19.

https://doi.org/10.1145/3318299.3318367. 